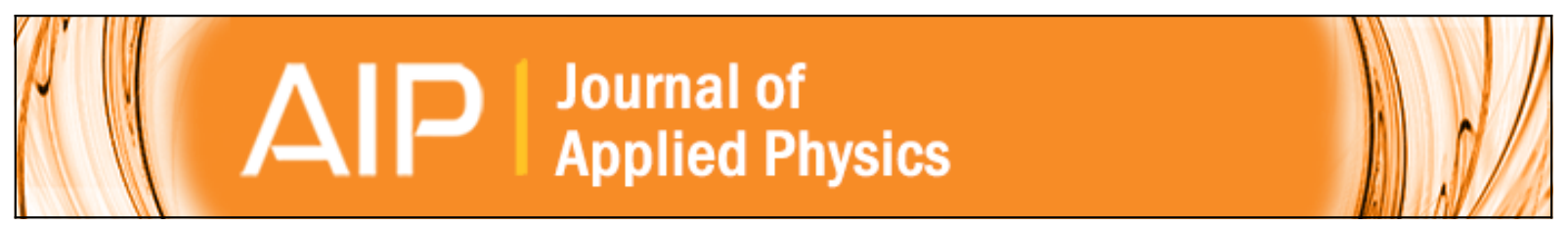

\title{
Optical properties and band bending of InGaAs/GaAsBi/InGaAs type-Il quantum well grown by gas source molecular beam epitaxy
}

Wenwu Pan, Liyao Zhang, Liang Zhu, Yaoyao Li, Xiren Chen, Xiaoyan Wu, Fan Zhang, Jun Shao, and Shumin Wang

Citation: Journal of Applied Physics 120, 105702 (2016); doi: 10.1063/1.4962288

View online: http://dx.doi.org/10.1063/1.4962288

View Table of Contents: http://scitation.aip.org/content/aip/journal/jap/120/10?ver=pdfcov

Published by the AIP Publishing

\section{Articles you may be interested in}

Study of molecular beam epitaxially grown InGaAsSbN/GaSb single quantum wells

J. Vac. Sci. Technol. B 29, 03C112 (2011); 10.1116/1.3555368

On overannealing of $\mathrm{Galn}(\mathrm{N}) \mathrm{As} / \mathrm{Ga}(\mathrm{N})$ As multiple quantum wells grown by molecular beam epitaxy

J. Appl. Phys. 102, 013504 (2007); 10.1063/1.2751483

Photoluminescence and photoreflectance study of InGaAs/AIAsSb quantum wells grown by molecular-beam epitaxy

J. Appl. Phys. 95, 1050 (2004); 10.1063/1.1637936

Effect of temperature on the optical properties of $\mathrm{GaAsSbN} / \mathrm{GaAs}$ single quantum wells grown by molecularbeam epitaxy

J. Appl. Phys. 93, 4475 (2003); 10.1063/1.1560574

Long wavelength GalnNAs/GaAs quantum-well heterostructures grown by solid-source molecular-beam epitaxy Appl. Phys. Lett. 77, 2189 (2000); 10.1063/1.1314295

\section{AlP Journal of Applied Physics \\ INTRODUCING INVITED PERSPECTIVES Ultrafast magnetism and $\mathrm{THz}$ spintronics Authors: Jakob Walowski and Markus Münzenberg}




\title{
Optical properties and band bending of InGaAs/GaAsBi/InGaAs type-II quantum well grown by gas source molecular beam epitaxy
}

\author{
Wenwu Pan, ${ }^{1,2}$ Liyao Zhang, ${ }^{1, a)}$ Liang Zhu, ${ }^{3}$ Yaoyao Li, ${ }^{1}$ Xiren Chen, ${ }^{3}$ Xiaoyan $\mathrm{Wu},{ }^{1,2}$ \\ Fan Zhang, ${ }^{1,4}$ Jun Shao, ${ }^{3}$ and Shumin Wang ${ }^{1,5, b}$ \\ ${ }^{1}$ State Key Laboratory of Functional Materials for Informatics, Shanghai Institute of Microsystem and \\ Information Technology, CAS, 865 Changning Road, Shanghai 200050, China \\ ${ }^{2}$ University of Chinese Academy of Sciences, Chinese Academy of Sciences, Beijing 100190, China \\ ${ }^{3}$ National Laboratory for Infrared Physics, Chinese Academy of Sciences, 500 Yutian Road, Shanghai 200083, \\ China \\ ${ }^{4}$ School of Physical Science and Technology, ShanghaiTech University, Shanghai 201210, China \\ ${ }^{5}$ Department of Microtechnology and Nanoscience, Chalmers University of Technology, Gothenburg 41296, \\ Sweden
}

(Received 22 June 2016; accepted 24 August 2016; published online 8 September 2016)

\begin{abstract}
Photoluminescence (PL) properties of $\mathrm{In}_{0.2} \mathrm{Ga}_{0.8} \mathrm{As} / \mathrm{GaAs}_{0.96} \mathrm{Bi}_{0.04} / \mathrm{In}_{0.2} \mathrm{Ga}_{0.8} \mathrm{As}$ quantum well (QW) grown on GaAs substrates by gas source molecular beam epitaxy were studied by varying excitation power and temperature, respectively. The type-II transition energy shifts from $1.149 \mathrm{eV}$ to $1.192 \mathrm{eV}$ when increasing the excitation power from $10 \mathrm{~mW}$ to $150 \mathrm{~mW}$ at $4.5 \mathrm{~K}$, which was ascribed to the band-bending effect. On the other hand, the type-II PL quenches quickly along with fast redshift with the increasing temperature due to the relaxation of the band bending caused by the thermal excitation process. An 8 band $\boldsymbol{k} \cdot \boldsymbol{p}$ model was used to analyze the electronic properties and the band-bending effect in the type-II QW. The calculated subband levels and transition energy fit well with the experiment results, and two thermal activation energies of $8.7 \mathrm{meV}$ and $50 \mathrm{meV}$, respectively, are deduced. Published by AIP Publishing. [http://dx.doi.org/10.1063/1.4962288]
\end{abstract}

\section{INTRODUCTION}

Recently, GaAsBi compounds have been considered to be promising materials for fiber-optic communication laser diodes with an efficient extension of light emitting wavelength, a relatively temperature-insensitive wavelength, and a reduction of Auger recombination. ${ }^{1-3}$ The incorporation of a small amount of $\mathrm{Bi}$ atoms into GaAs strongly reduces the band-gap energy $(84-91 \mathrm{meV} / \% \mathrm{Bi})^{4-6}$ and, in addition, decreases the temperature coefficient of the band gap from $0.45 \mathrm{meV} / \mathrm{K}$ to about $0.15 \mathrm{meV} / \mathrm{K}^{2,7}$ For GaAsBi with Bi contents of above $10 \%$, the spin-orbit splitting energy $\left(\Delta_{\mathrm{SO}}\right)$ becomes larger than the band gap, and thus, the hot-holegenerating Auger recombination and the inter-valence band absorption processes are expected to be suppressed. ${ }^{1,8,9}$ So far, light emitting diodes (LEDs) with $\mathrm{GaAs}_{0.94} \mathrm{Bi}_{0.06}$ as active regions have shown a good room-temperature electroluminescence at about $1.20 \mu \mathrm{m} .{ }^{10}$ Meanwhile, the electrically pumped GaAs-based lasers with three $\mathrm{GaAs}_{0.94} \mathrm{Bi}_{0.06}$ active quantum wells (QWs) have been fabricated successfully with the lasing wavelength up to $1.06 \mu \mathrm{m} .{ }^{11}$ However, the room temperature emission at longer wavelengths by using conventional type-I GaAsBi QWs grown on GaAs is rather limited due primarily to the difficulty in incorporating a high content of $\mathrm{Bi}(>6 \%)$ and simultaneously retaining the high optical quality. ${ }^{12-14}$ The reported maximum growth temperature for fabricating device in the molecular beam epitaxy (MBE) is about $380^{\circ} \mathrm{C}$, and only $4 \%-6 \% \mathrm{Bi}$ is incorporated in the GaAsBi active layer. ${ }^{2,11,15,16}$

\footnotetext{
${ }^{\text {a)} E-m a i l: ~ l y z h a n g @ m a i l . s i m . a c . c n ~}$

${ }^{b)}$ E-mail: Shumin@mail.sim.ac.cn
}

It is well known that the type-II band structure allows a QW with less lattice mismatch to achieve longer emission wavelength and the type-II heterostructures employing the $\operatorname{InGaAs}(\mathrm{N}) / \mathrm{GaAsSb}$ system on GaAs have been previously demonstrated to be promising for laser emitting at $1.55 \mu \mathrm{m} .{ }^{17,18}$ Moreover, a type-II structure enables the energy band bending effect, which induces a blue shift in photoluminescence (PL) when the excitation power increases. Generally speaking, the mechanism of this effect has been discussed in terms of a triangular potential model in which the photogenerated electrons and holes form a dipole layer, creating a triangular-like potential at the interface, ${ }^{19}$ while Jo et al. ${ }^{20}$ found that the dominant contribution to the blueshift originates from the variation of the QW energy level after performing a self-consistent approach including the excitonic effect. On the other hand, the thermal escape of electrons from the GaAs well into the GaAsSb barrier is responsible for the PL quenching observed in the temperature dependent PL spectra of type-II GaAsSb/GaAs multiple quantum wells. ${ }^{19}$

Recently, we have grown the new type II InGaAs/ GaAsBi/InGaAs QW by gas source MBE. ${ }^{21}$ The emission wavelength was extended to $1070 \mathrm{~nm}$ at $4.5 \mathrm{~K}$, longer than $943 \mathrm{~nm}$ and $1006 \mathrm{~nm}$ from the corresponding type-I InGaAs and GaAsBi QW, respectively. Our experimental and theoretical results show that this new kind of heterostructure may provide an interesting alternative to the type-I GaAsBi material for a near infrared generation. However, the spectroscopy property of this system has not been deeply explored. In this paper, the PL properties of the type II InGaAs/ GaAsBi QW have been investigated by varying excitation power and temperature, respectively. Meanwhile, an 8 band 
$\boldsymbol{k} \boldsymbol{p}$ model combined with space charge effects was used to analyze the electronic properties and the bending effects observed in the experiment.

\section{EXPERIMENTS}

The type-II structure, which consists of $100 \mathrm{~nm} \mathrm{GaAs}$ buffer $/ 8.5 \mathrm{~nm} \quad \mathrm{In}_{0.2} \mathrm{Ga}_{0.8} \mathrm{As} / 7.8 \mathrm{~nm} \quad \mathrm{GaAs}_{0.96} \mathrm{Bi}_{0.04} / 8.5 \mathrm{~nm}$ $\mathrm{In}_{0.2} \mathrm{Ga}_{0.8} \mathrm{As} / 100 \mathrm{~nm}$ GaAs cap layers, was grown on semiinsulating GaAs (001) substrates using a V90 GSMBE system equipped with effusion cells for $\mathrm{In}, \mathrm{Ga}$, and $\mathrm{Bi}$. $\mathrm{As}_{2}$ was cracked from arsine at $1000^{\circ} \mathrm{C}$, its flux was controlled by regulating the gas pressure in the gas line, and the substrate temperature was measured by a thermocouple. For the growth of $\mathrm{InGaAs} / \mathrm{GaAsBi} / \mathrm{InGaAs}$ active region, the substrate temperature was decreased to $410^{\circ} \mathrm{C}$. No dislocations were observed during the high-resolution cross section TEM characterization, indicating such thick three-layer wells grown at low temperature remain elastically strain, and the details of the growth condition as well as the composition determination can be found elsewhere. ${ }^{21}$ A diode pumped solid-state (DPSS) laser $(\lambda=639 \mathrm{~nm})$ was used as the excitation source.

\section{RESULTS AND DISCUSSION}

\section{A. Excitation-dependent PL}

Figure 1 shows the $4.5 \mathrm{~K}$ PL spectra of the InGaAs/ GaAsBi/InGaAs QW under different excitation intensities. Two peaks between $1.10 \mathrm{eV}$ and $1.35 \mathrm{eV}$ were observed. One peak located at $1.313 \mathrm{eV}$, labeled as $\mathrm{P} 1$, corresponds to the type-I transition in InGaAs layers, while the other peak at a lower energy with a much broader and intense feature, labeled as P2, originates from the type-II transition between the electrons in the InGaAs conduction band and holes confined in the GaAsBi valence band. When the incorporated $\mathrm{Bi}$ content reaches up to $4 \%$, the valence band offset (VBO) of $\mathrm{GaAsBi} / \mathrm{InGaAs}$ increases to such that most holes are confined in the GaAsBi layer causing the type-II radiative

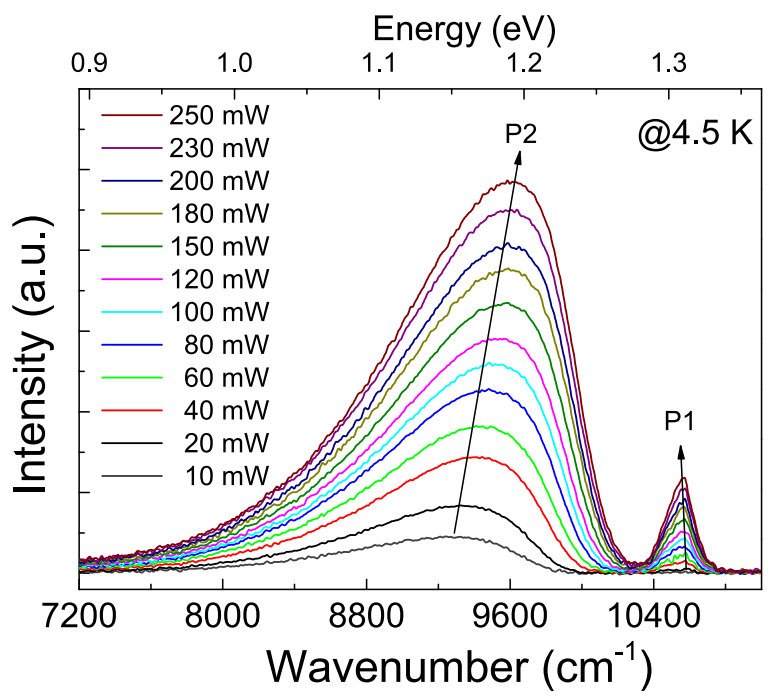

FIG. 1. The 4.5 K PL spectra of $\operatorname{In}_{0.2} \mathrm{Ga}_{0.8} \mathrm{As} / \mathrm{GaAs}_{0.96} \mathrm{Bi}_{0.04} / \operatorname{In}_{0.2} \mathrm{Ga}_{0.8} \mathrm{As}$ QW under different excitation powers. recombination. From the PL spectrum excited under a low power of $10 \mathrm{~mW}$, it can be obtained that the energy difference between the peak $\mathrm{P} 1(1.315 \mathrm{eV})$ and $\mathrm{P} 2(1.149 \mathrm{eV})$ is about $166 \mathrm{meV}$, which is close to the estimated VBO of $169 \mathrm{meV}$ for $\mathrm{In}_{0.2} \mathrm{Ga}_{0.8} \mathrm{As} / \mathrm{GaAs}_{0.96} \mathrm{Bi}_{0.04}$ heterojunction. ${ }^{21}$ The FWHM of the P2 are about $132 \mathrm{meV}$, which is much broader than the P1 of $25 \mathrm{meV}$. Such a broadening effect is related to the non-uniform distribution of Bi and/or Bi clusters in the GaAsBi layer. ${ }^{22}$

Surprisingly, we observed a giant blueshift under a moderate excitation intensity. As shown in Fig. 2, the PL peak energy of P2 has a shift of about $41 \mathrm{meV}$, and its FWHM increases when the pumping intensity changes from 10 to $200 \mathrm{~mW}$, while those of P1 are almost not affected. The blueshift rate decreases, and its value as well as the FWHM eventually saturates to be about $1.192 \mathrm{eV}$ and $143 \mathrm{meV}$, respectively, when the excitation power increases beyond $150 \mathrm{~mW}$. This peculiar behavior cannot be ascribed to radiation heating, which should cause a shrinkage in the band gap. Also, the giant blueshift is hard to be assigned to the state filling of the localized states from the interface roughness or alloy potential fluctuations since it can be easily saturated at lower excitation power because of the low density of the localized states in comparison to the density of states for the QW ground states. In GaAsBi alloys, the carrier localization is a well-known phenomenon, and the so called S-shape behavior can be seen in the temperature dependent PL under the low excitation conditions. ${ }^{23,24}$ In addition, Baranowski et al. ${ }^{25,26}$ found that the PL kinetics are strongly influenced by the band bending effects as well as the carrier localization effects in type II GaAsSb/GaAs QWs using a time-resolved PL spectroscopy, suggesting that further investigations of the carrier localization phenomena in this InGaAs/GaAsBi QW are needed to evaluate its quality and perspectives for device applications. We believe that the underlying mechanism of the observations results from the band bending effect, which is intrinsic for the type-II band alignment of $\mathrm{In}_{0.2} \mathrm{Ga}_{0.8} \mathrm{As} /$ $\mathrm{GaAs}_{0.96} \mathrm{Bi}_{0.04}$ heterojunction, similar to the cases of GaAs/ $\mathrm{GaAsSb}^{19}$ and $\mathrm{InGaAs} / \mathrm{GaAsSb}{ }^{27}$ It is commonly accepted that the band-bending effect is caused by the spatial separation of the photo-excited or electrically pumped carriers in a

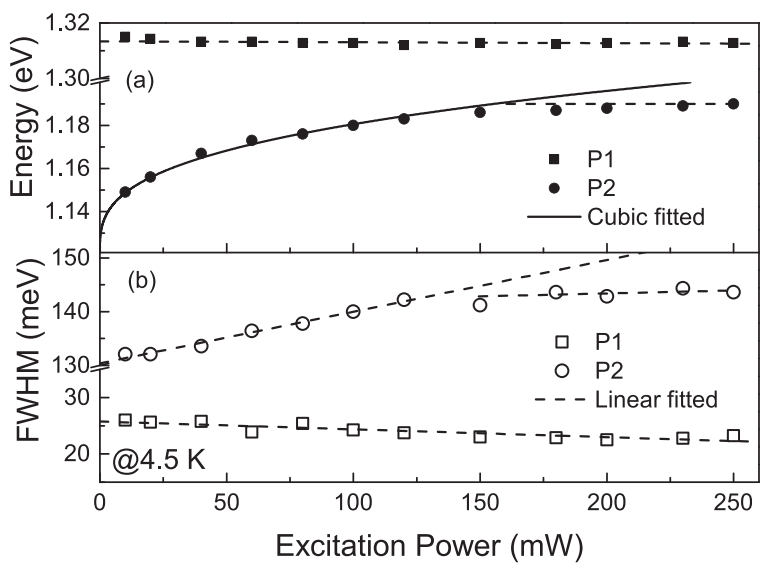

FIG. 2. The dependence of the peak energy (a) as well as the FWHM (b) of $\mathrm{P} 1$ and $\mathrm{P} 2$ on excitation power. A strong blueshift and saturation of $\mathrm{P} 2$ with the increase of excitation power are observed. 


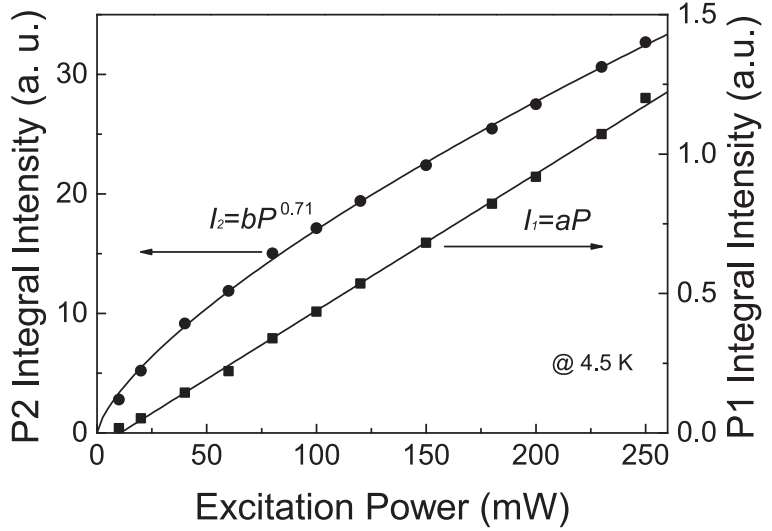

FIG. 3. The dependence of integral PL intensity of P1 and P2 on pumping power.

type-II QW, in which the type-II transition energy is expected to increase proportionally with the third root of the excitation density due to the steepness raising of a triangular-like confining potential. ${ }^{19}$ For the $\mathrm{In}_{0.2} \mathrm{Ga}_{0.8} \mathrm{As} /$ GaAsBi type-II QW, electrons are confined in the InGaAs layer, and holes are localized in the GaAsBi layer. Indeed, a cubic curve as shown in Fig. 2(a) fits well with the experimental results with an excitation power below $150 \mathrm{~mW}$. When the excitation power exceeds $150 \mathrm{~mW}$, the energy blueshift as well as the FWHM broadening of P2 stops. This behavior can also be explained by the band bending model. Due to the band bending, there will be a potential barrier for electrons and holes in the GaAsBi and InGaAs layer, respectively. If the band bending is large enough, the barrier is able to prevent the photo-excited carriers from transferring from the high-energy band to the low-energy band, and the blueshift therefore saturates.

Figure 3 displays the dependence of integral PL intensities of $\mathrm{P} 1$ and $\mathrm{P} 2$, labeled as $I_{1}$ and $I_{2}$, on the pumping power $P$, respectively. We can see that the $I_{1}$ increases linearly while the $I_{2}$ shows a sublinear behavior with the increasing of $P$, respectively. For the semiconductor with a low background carrier density, the relationship between $I$ and $P$ can be reduced to a simple power law as $I \propto P^{\mathrm{m}}$, where $\mathrm{m}=1,2$, or $2 / 3$ corresponds to radiative, Shockley-Read-Hall nonradiative, and Auger recombination, respectively. ${ }^{28}$ The slope of the plot in Fig. 3 is fitted as $m=1$ and 0.71 for P1 and $\mathrm{P} 2$, respectively, which indicates that the dominant carrier recombination mechanisms for the InGaAs/GaABi type II QW mainly include radiative and Auger recombination at $4.5 \mathrm{~K}$.

\section{B. Temperature-dependent PL}

Figure 4 shows the temperature dependent PL spectra with a fixed excitation of $120 \mathrm{~mW}$ measured at temperature varying from $4.5 \mathrm{~K}$ to $290 \mathrm{~K}$. We can see that both the peak energy and intensity of P1 and P2 decrease with the increasing temperature. Especially for P2, the PL intensity decreases gradually with the increasing temperature from $4.5 \mathrm{~K}$ to $130 \mathrm{~K}$ and then quenches quickly when temperature increases beyond $160 \mathrm{~K}$. In addition, compared with the $41 \mathrm{meV}$ redshift of P1, a larger redshift of P2, i.e., $84 \mathrm{meV}$ as

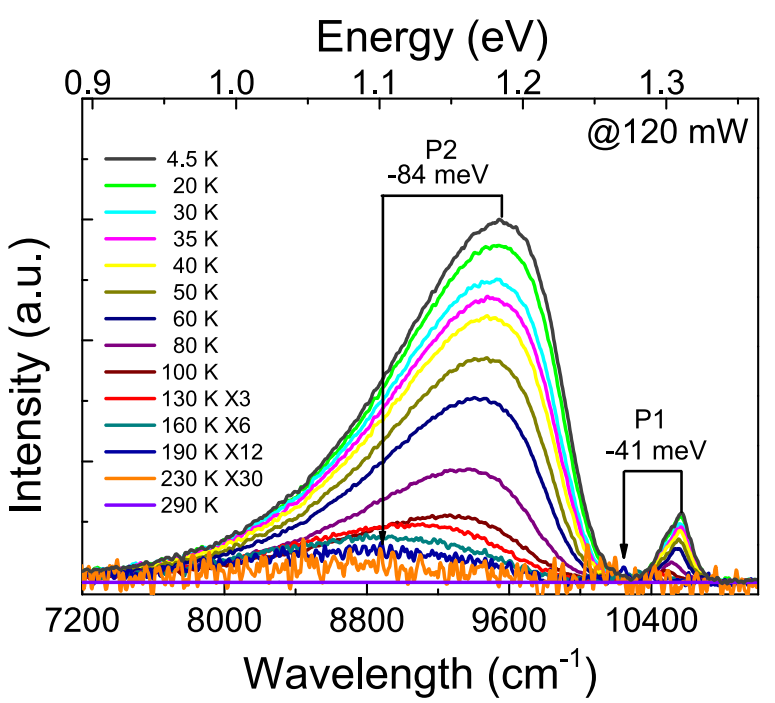

FIG. 4. The temperature dependent PL spectra of InGaAs/GaAsBi/InGaAs QW measured with a $120 \mathrm{~mW}$ excitation power.

illustrated in Fig. 4, was found when increasing the temperature from $4.5 \mathrm{~K}$ to $190 \mathrm{~K}$. The energies and FWHMs of P1 and $\mathrm{P} 2$ are summarized in Fig. 5, and no obvious S-shape behaviors of the two peaks are observed at a low temperature, indicating the carrier localization effect maybe saturated at this excitation conditions. For the peak energy of P1, it can be analyzed using the Varshni relationship ${ }^{29}$ of

$$
\mathrm{E}=\mathrm{E}_{0}-\alpha \mathrm{T}^{2} /(\beta+T),
$$

where $E_{0}$ is the fundamental excitonic transition energy at absolute zero temperature, $\alpha$ and $\beta$ are the adjustable parameters. The constant $\alpha$ is related to the electron-phonon interaction, and $\beta$ is closely related to the Debye temperature. As shown in Fig. 5(a), for P1, the best fit to the experimental data is obtained with $\alpha=0.486 \mathrm{meV} / \mathrm{K}, \beta=197 \mathrm{~K}$, and $\mathrm{E}_{0}=1.313 \mathrm{eV}$. The values of $\alpha$ and $\beta$ are very close to those of bulk $\mathrm{In}_{0.2} \mathrm{Ga}_{0.8} \mathrm{As} .{ }^{30}$ However, for P2, with temperature increasing from $60 \mathrm{~K}$ to $160 \mathrm{~K}$, a much faster redshift was observed. The FWHM of P2 remains at around $140 \mathrm{meV}$ for $T<60 \mathrm{~K}$ and almost increases linearly with temperature for $60 \mathrm{~K}<T<160 \mathrm{~K}$, while that of P1 is almost not affected in

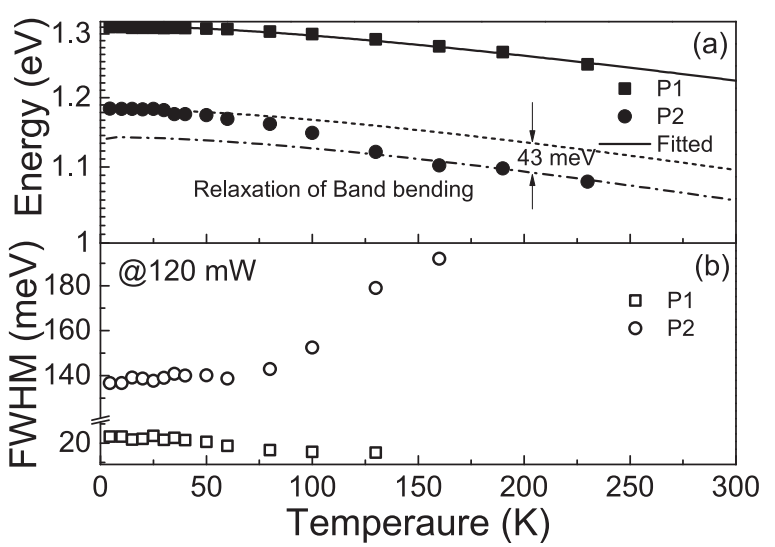

FIG. 5. Dependence of the PL peak energy as well as FWHM of P1 and P2 on temperature. 
the temperature range up to $130 \mathrm{~K}$ as shown in Fig. 5(b). The redshift cannot be explained by the thermal population of the electron and hole states with high- $k$ values, which have a high probability of radiative recombination since the PL maxima should shift to higher-photon energies relative to the effective band-gap energy. This behavior is also related to the band bending effects in type-II QWs, where the quantum confinement becomes less pronounced with the increasing temperature. At a high temperature, the ability of the triangular-like potential to confine carriers is reduced since the photo-excited carriers can be thermally excited to escape from the InGaAs/GaAsBi interface. The relaxation of the band bending leads to a much faster reduction of effective band gap in the type-II QW than that of in bulk or a normal type-I QW like InGaAs. As shown in Fig. 5, about a $43 \mathrm{meV}$ redshift contribution by band bending relaxation is deduced by comparing the temperature dependent behaviors between $\mathrm{P} 1$ and $\mathrm{P} 2$. Thus, the flat-band transition energy of P2 is estimated to be $1.142 \mathrm{meV}$ at $4.5 \mathrm{~K}$. In Fig. 6, we display the Arrhenius plots of the intensity as well as the integral intensity of the P1 and P2, respectively. The solid lines are calculated by the expression

$$
I=I_{0} /\left(1+C_{1} e^{-E_{1} / k T}+C_{2} e^{-E_{2} / k T}\right),
$$

where $I_{0}$ is the integral PL intensity at $0 \mathrm{~K}, C_{1}$ and $C_{2}$ are constants, and $E_{1}$ and $E_{2}$ are the thermal activation energies. For the integral intensity of P2, as depicted in Fig. 6, the obtained $E_{1}$ and $E_{2}$ values of the PL quenching are $8.7 \mathrm{meV}$ and $50.0 \mathrm{meV}$, respectively. It should be noted that the deduced thermal activation energy of $E_{1}$ is sensitive to the accuracy of PL data obtained in the low temperature range, while $E_{2}$ is sensitive to the certainty of the PL data measured at a relatively high temperature. Thus, a similar value of $E_{1}$, i.e., $8.4 \mathrm{meV}$, is deduced from the temperature dependence of the integral intensity of P1 as the line shapes of P1 measured at $\mathrm{T}<100 \mathrm{~K}$ are fixed as shown in Fig. 4. However, the weak PL features of P1 measured beyond $100 \mathrm{~K}$ lead to a large deviation when deducing the thermal activation energy of $E_{2}$. The origination of the two thermal activation energies of $E_{1}$ and $E_{2}$ will be discussed later.

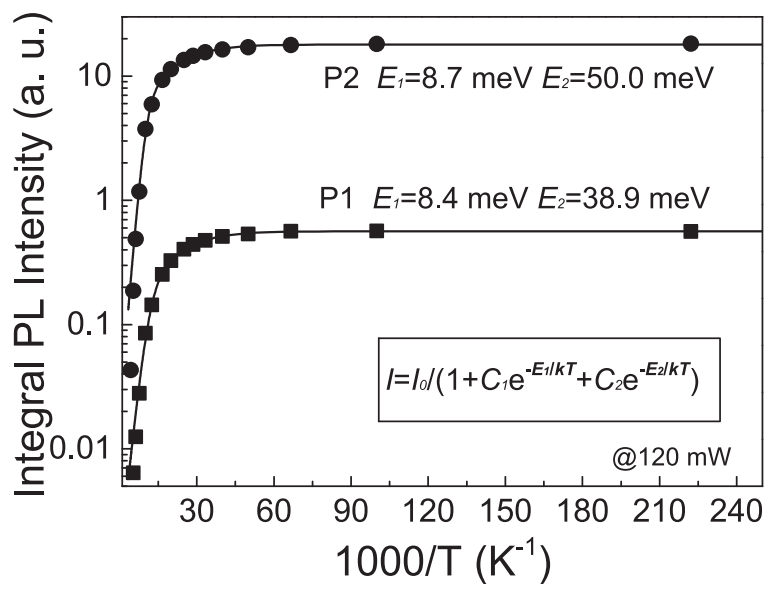

FIG. 6. Logarithm of the PL and PL integral intensity of P1 and P2 versus reciprocal temperature together with the respective activation energy fit.

\section{Electronic properties}

In order to interpret the band bending effect and the PL quench observed in our experimental results, it is necessary to calculate the energy levels and wave-functions under different injection levels for the structure described above. Thus, a semi-quantitative analysis of the band bending based on the 8 band $\boldsymbol{k}$. model has been performed. In the calculations reported in this paper, band offset ratios of 60:40 between the strained InGaAs and GaAs and 24:76 between the strained GaAsBi and GaAs were used, respectively. ${ }^{6,31}$ The transformation of GaAsBi and $\mathrm{In}_{0.2} \mathrm{Ga}_{0.8}$ As to form a type-II configuration from a type-I configuration is estimated to occur near a Bi concentration of $1.3 \%$. A schematic diagram of the conduction and valence bands in the active region of our structure is shown in Fig. 7(a), where the valence band edge of GaAs is taken to be zero energy. The GaAsBi hole QW, which is $7.8 \mathrm{~nm}$ thick and has a nominal Bi content of $4 \%$, is sandwiched between the two $8.5 \mathrm{~nm}$ $\mathrm{In}_{0.2} \mathrm{Ga}_{0.8}$ As electron QWs. We have used the material parameters $^{21}$ to solve Schrödinger's equation for flat band conditions as well as for carrier injection by performing selfconsistent calculations. The calculated energy levels within the schematic band diagram and the selected envelope wave functions are shown in Fig. 7.

For the flat band, as shown in Fig. 7(a), in the conduction band, the thin GaAsBi layer allows the electron wavefunctions in the InGaAs QWs to couple, resulting in two coupled solutions, symmetric $(\mathrm{e} 1 \mathrm{~S}=1.403 \mathrm{eV})$ and antisymmetric $(\mathrm{e} 1 \mathrm{~A}=1.408 \mathrm{eV})$, which are confined in the InGaAs QWs. The first electron excited sate $(\mathrm{e} 2=1.465 \mathrm{eV})$ shows localized features in GaAsBi layer. In the valence band, the heavy and light hole solutions are strongly confined to the GaAsBi layer. The calculations show that, due to the difference in the heavy and light hole masses, two heavy hole energy levels $(\mathrm{HH} 1=0.239 \mathrm{eV} ; \mathrm{HH} 2=0.203 \mathrm{eV})$ occur in the GaAsBi QW at lower energy than the first light hole solution $(\mathrm{LH} 1=0.178 \mathrm{eV})$. In the $\mathrm{InGaAs}$ layer, the first heavy hole solution $(\mathrm{hh} 1=0.072 \mathrm{eV})$ is confined in the

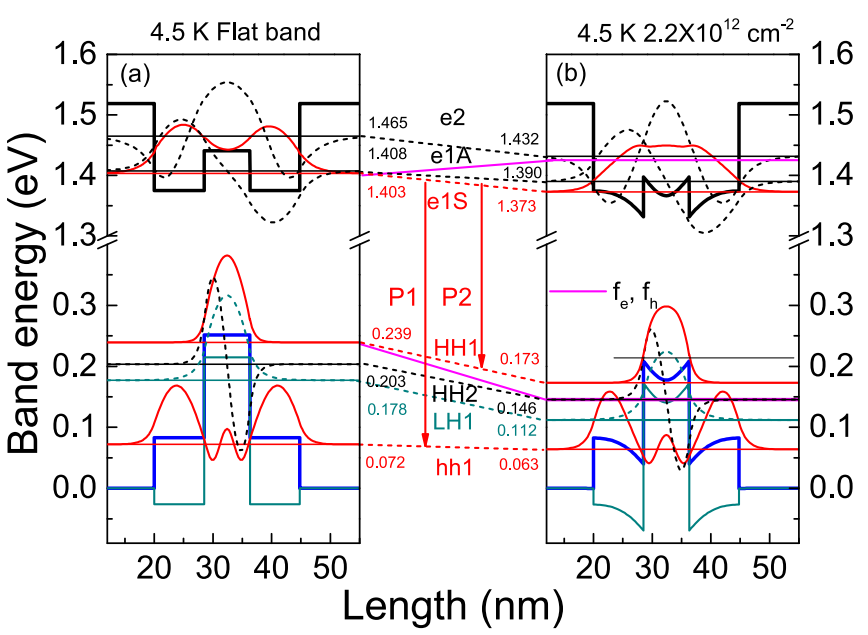

FIG. 7. The $4.5 \mathrm{~K}$ bulk bandedge profiles and selected wave functions calculated for flat band (a) and injecting e-h density $2.2 \times 10^{12} \mathrm{~cm}^{-2}$ (b). Legends for wave functions and the corresponding electron and hole subband energy levels are depicted in the figure. 
InGaAs QWs. The type-I transition energy between e1S and hh1 with an overlap of $93 \%$ is $1.331 \mathrm{eV}$. The $29 \%$ overlap of the wave-functions enables the type-II transitions between the e1S and the HH1 with a transition energy of $1.164 \mathrm{eV}$. Both the transition energy values are about $20 \mathrm{meV}$ larger than the experimental observation discussed previously, i.e., $1.313 \mathrm{eV}$ and $1.142 \mathrm{eV}$, respectively. The deviation may result from the underestimation of about $2 \%$ In content in the InGaAs layers.

For the band bending, we consider the change of the non-equilibrium carrier density due to photo-excitation. As shown in Fig. 7(b), when the injected e-h pair density increases to $2.2 \times 10^{12} \mathrm{~cm}^{-2}$, two approximately triangular wells are produced due to the strong band bending near the InGaAs/GaAsBi interface. Meanwhile, the quasi-Fermi level of the photo-generated electrons $\left(f_{e}\right)$ has not exceed the e2, while that of holes $\left(f_{h}\right)$ is just reaching to the $2 \mathrm{HH}$ subband level, indicating that the photo-excited electrons and holes are majorly accumulated in the InGaAs and GaAsBi, respectively, and the band bending will be triggered when there is a further increase in the e-h density. Due to the band bending effect, both the electron and the hole subband levels decrease. However, the HH1 subband level shift is larger than the e1S subband level, and as a result, the transition energy of P2 shows a blueshift about $36 \mathrm{meV}$. Meanwhile, it should be noted that, with the increasing of the band-bending effects, the wave functions of the e1S and $1 \mathrm{HH}$ states will penetrate further into each other, and thus, the ground electron and hole wavefunctions become less spatially separated and the structure tends to be type-I. This is the reason that the blueshift rate of P2 will be slowed down and the peak energy of $\mathrm{P} 2$ will be saturated at a high excitation power.

It should be noted that the band bending model based on the $\boldsymbol{k} \cdot \boldsymbol{p}$ method works in the case of low temperature since the thermal activation process of the injected carriers is not considered. The temperature dependent PL behavior summarized in Fig. 4 shows that the band bending can be relaxed when the temperature increases up to $160 \mathrm{~K}$ due to the thermal activation of carriers. In addition, two activation energies of $E_{1}=8.7 \mathrm{meV}$ and $E_{2}=50.0 \mathrm{meV}$, which are deduced from the temperature dependent integral intensity of $\mathrm{P} 2$, are close to the value of the energy difference between e1S and the e $1 \mathrm{~A}(5 \mathrm{meV})$, and the first delocalized state e $2(62 \mathrm{meV})$ as indicated in Fig. 7. This implies that the thermal escape of electrons from the ground state localized near the InGaAs/ GaAsBi interface into the whole QW is responsible for the PL quenching in the InGaAs/GaAsBi/InGaAs QW near room temperature. Thus, we can expect that increasing In content should be useful for the room temperature PL emission of the InGaAs/GaAsBi type II QW due to the improvement of electron confinement. Furthermore, for the fact that no $\mathrm{PL}$ at room temperature was observed in the type II InGaAs/GaAsBi QW under the excitation of $120 \mathrm{~mW}$, a great number of non-radiative centers, produced during the low temperature growth of the active region, are also probably responsible for this issue. Further optimization of the growth conditions, such as increasing the growth temperature of the two InGaAs layers, is needed to reduce the non- radiative centers and improve the material quality for device applications.

Figure 8(a) shows the subband energies as well as quasiFermi levels as a function of the injected e-h sheet density based on the calculation. Also, the calculated energy shifts of $\mathrm{P} 1$ and $\mathrm{P} 2$ are also shown in Fig. 8(b). The blueshift of P2 is about $36 \mathrm{meV}$ and $40 \mathrm{meV}$ when the $\mathrm{f}_{\mathrm{h}}$ and the $\mathrm{f}_{\mathrm{e}}$ just exceed the $\mathrm{HH} 2$ and e1A subbands, respectively. With a further increase of injecting density to $4 \times 10^{12} \mathrm{~cm}^{-2}$ as shown in Fig. 8, the blueshift of the transition energy P2 due to the strong band bending tends to be saturate at $48 \mathrm{meV}$, which is close to the experiment observation of $50 \mathrm{meV}$. For the type I transition $\mathrm{P} 1$, the calculation results suggest a redshift due to the band bending effect, which is not consistent with the excitation power dependent PL spectra as shown in Fig. 1. As reported in Ref. 21, the Bi segregation in the growth direction takes place, and the maximum $\mathrm{Bi}$ concentration appears at much closer to the upper $\mathrm{InGaAs} / \mathrm{GaAsBi}$ interface. This is caused by the relatively high growth temperature for $\mathrm{GaAsBi}$ since the Bi surface segregation can be enhanced with the increasing growth temperature. ${ }^{32}$ The transition of P1 we observed most likely originates from the lower InGaAs layer where the possibility of achieving the type II band alignment in the lower $\mathrm{GaAsBi} / \mathrm{InGaAs}$ interface is small, and thus, the band bending effect is negligible. If the $\mathrm{P} 1$ originates from the upper type II InGaAs/GaAsBi quantum well, the relaxation of the band bending will slow down the redshift of P1, while this was not observed in the temperature dependent PL, and further work is required to confirm it.

\section{SUMMARY}

In summary, we have reported several peculiar behaviors in the PL spectra of InGaAs/GaAsBi type II QWs related to the band bending caused by the dipole formation by

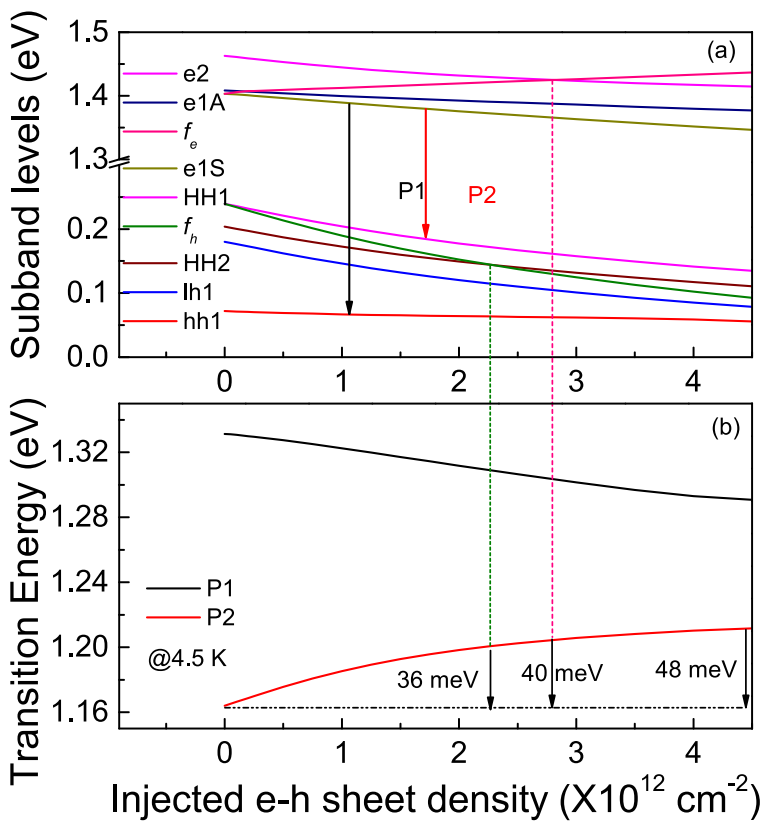

FIG. 8. The calculated changes of subband energy levels and the quasiFermi levels with varying e-h sheet density injected in the InGaAs/GaAsBi/ InGaAs QW based on the $8 \boldsymbol{k} \cdot \boldsymbol{p}$ model (a). The calculated transition energies of P1 and P2 are also plotted (b). 
separation of electrons and holes, including a giant blueshift, a saturation effect, and the relaxation of band bending due to the thermal excitation process. The experimental results are well explained by the $\boldsymbol{k} \cdot \boldsymbol{p}$ model. Our results are instructive for understanding the optical properties in the InGaAs/ GaAsBi type-II semiconductor heterostructures.

\section{ACKNOWLEDGMENTS}

The authors wish to acknowledge the financial support of the National Basic Research Program of China (Grant No. 2014CB643902), the Key Program of Natural Science Foundation of China (Grant No. 61334004), the Natural Science Foundation of China (Grant No. 61404152), the "Strategic Priority Research Program" of the Chinese Academy of Sciences (Grant No. XDA5-1), the foundation of National Laboratory for Infrared Physics, the Key Research Program of the Chinese Academy of Sciences (Grant No. KGZD-EW-804), and the Creative Research Group Project of Natural Science Foundation of China (Grant No. 61321492).

${ }^{1}$ C. A. Broderick, M. Usman, S. J. Sweeney, and E. P. O'Reilly, Semicond. Sci. Technol. 27, 094011 (2012).

${ }^{2}$ T. Fuyuki, K. Yoshida, R. Yoshioka, and M. Yoshimoto, Appl. Phys. Express 7, 082101 (2014).

${ }^{3}$ S. J. Sweeney and S. R. Jin, J. Appl. Phys. 113, 043110 (2013).

${ }^{4}$ S. Tixier, M. Adamcyk, T. Tiedje, S. Francoeur, A. Mascarenhas, P. Wei, and F. Schiettekatte, Appl. Phys. Lett. 82, 2245 (2003).

${ }^{5}$ S. Francoeur, M. J. Seong, A. Mascarenhas, S. Tixier, M. Adamcyk, and T. Tiedje, Appl. Phys. Lett. 82, 3874 (2003).

${ }^{6}$ M. P. Polak, P. Scharoch, and R. Kudrawiec, Semicond. Sci. Technol. 30, 094001 (2015).

${ }^{7}$ J. Yoshida, T. Kita, O. Wada, and K. Oe, Jpn. J. Appl. Phys., Part 142 , 371 (2003).

${ }^{8}$ B. Fluegel, S. Francoeur, A. Mascarenhas, S. Tixier, E. C. Young, and T. Tiedje, Phys. Rev. Lett. 97, 067205 (2006).

${ }^{9}$ K. Alberi, O. D. Dubon, W. Walukiewicz, K. M. Yu, K. Bertulis, and A. Krotkus, Appl. Phys. Lett. 91, 051909 (2007).

${ }^{10}$ R. D. Richards, A. R. Mohmad, J. P. R. David, C. J. Hunter, and F. Bastiman, IET Optoelectron. 10, 34 (2016).
${ }^{11}$ R. Butkute, A. Geizutis, V. Pacebutas, B. Cechavicius, V. Bukauskas, R. Kundrotas, P. Ludewig, K. Volz, and A. Krotkus, Electron. Lett. 50, 1155 (2014).

${ }^{12}$ M. Yoshimoto, S. Murata, A. Chayahara, Y. Horino, J. Saraie, and K. Oe, Jpn. J. Appl. Phys., Part 2 42, L1235 (2003).

${ }^{13}$ V. Bahrami-Yekta, T. Tiedje, and M. Masnadi-Shirazi, Semicond. Sci. Technol. 30, 094007 (2015).

${ }^{14}$ W. Bennarndt, G. Boehm, and M. C. Amann, J. Cryst. Growth 436, 56 (2016).

${ }^{15}$ C. J. Hunter, F. Bastiman, A. R. Mohmad, R. Richards, J. S. Ng, S. J. Sweeney, and J. P. R. David, IEEE Photonics Technol. Lett. 24, 2191 (2012).

${ }^{16}$ T. Fuyuki, R. Yoshioka, K. Yoshida, and M. Yoshimoto, Appl. Phys. Lett. 103, 202105 (2013).

${ }^{17}$ I. Vurgaftman, J. R. Meyer, N. Tansu, and L. J. Mawst, Appl. Phys. Lett. 83, 2742 (2003).

${ }^{18}$ B. Chen, A. L. Holmes, V. Khalfin, I. Kudryashov, and B. M. Onat, Laser Technology for Defense and Security VIII 8381, 83810F (2012).

${ }^{19}$ Y. S. Chiu, M. H. Ya, W. S. Su, and Y. F. Chen, J. Appl. Phys. 92, 5810 (2002).

${ }^{20}$ M. Jo, M. Sato, S. Miyamura, H. Sasakura, H. Kumano, and I. Suemune, Nanoscale Res. Lett. 7, 1 (2012).

${ }^{21}$ See https://arxiv.org/abs/1606.04657 for information about the structural properties of the sample.

${ }^{22}$ A. R. Mohmad, F. Bastiman, C. J. Hunter, J. S. Ng, S. J. Sweeney, and J. P. R. David, Appl. Phys. Lett. 99, 042107 (2011).

${ }^{23}$ R. Kudrawiec, M. Syperek, P. Poloczek, J. Misiewicz, R. H. Mari, M. Shafi, M. Henini, Y. G. Gobato, S. V. Novikov, J. Ibanez, M. Schmidbauer, and S. I. Molina, J. Appl. Phys. 106, 023518 (2009).

${ }^{24}$ S. Imhof, A. Thranhardt, A. Chernikov, M. Koch, N. S. Koster, K. Kolata, S. Chatterjee, S. W. Koch, X. F. Lu, S. R. Johnson, D. A. Beaton, T. Tiedje, and O. Rubel, Appl. Phys. Lett. 96, 131115 (2010).

${ }^{25}$ M. Baranowski, M. Syperek, R. Kudrawiec, J. Misiewicz, J. A. Gupta, X. Wu, and R. Wang, Appl. Phys. Lett. 98, 061910 (2011).

${ }^{26}$ M. Baranowski, M. Syperek, R. Kudrawiec, J. Misiewicz, J. A. Gupta, X. Wu, and R. Wang, J. Phys.: Condens. Matter 24, 185801 (2012).

${ }^{27}$ J. Hu, X. G. Xu, J. A. H. Stotz, S. P. Watkins, A. E. Curzon, M. L. W. Thewalt, N. Matine, and C. R. Bolognesi, Appl. Phys. Lett. 73, 2799 (1998).

${ }^{28}$ I. P. Seetoh, C. B. Soh, E. A. Fitzgerald, and S. J. Chua, Appl. Phys. Lett. 102, 101112 (2013).

${ }^{29}$ Y. P. Varshni, Physica 34, 149 (1967).

${ }^{30}$ I. Vurgaftman, J. R. Meyer, and L. R. Ram-Mohan, J. Appl. Phys. 89, 5815 (2001).

${ }^{31}$ J. P. Reithmaier, R. Hoüger, H. Riechert, A. Heberle, G. Abstreiter, and G. Weimann, Appl. Phys. Lett. 56, 536 (1990).

${ }^{32}$ E. Luna, M. Wu, M. Hanke, J. Puustinen, M. Guina, and A. Trampert, Nanotechnology 27, 325603 (2016). 